\title{
Rapid Detection of Quinolone Resistance Mutations in gyrA of Helicobacter pylori by Real-Time PCR
}

\author{
Franziska Haumaier ${ }^{1,2,+}$, Anna Schneider-Fuchs ${ }^{2,+}$, Steffen Backert ${ }^{3}\left(\mathbb{D}\right.$, Michael Vieth $^{2,4}$, William Sterlacci ${ }^{2,4, *}$ \\ and Birgitta M. Wöhrl $1,5, *$ (D)
}

1 Lehrstuhl Biochemie IV-Biophysikalische Chemie, Universität Bayreuth, Universitätsstraße 30, 95447 Bayreuth, Germany; franzihaumaier@googlemail.com

2 Institut für Pathologie, Klinikum Bayreuth, Preuschwitzer Straße 101, 95445 Bayreuth, Germany; anna.schneider-fuchs@klinikum-bayreuth.de (A.S.-F.); Vieth.LKPathol@uni-bayreuth.de (M.V.)

3 Department Biologie, Lehrstuhl Für Mikrobiologie, Friedrich-Alexander Universität Erlangen-Nürnberg, Staudtstraße 5, 91058 Erlangen, Germany; steffen.backert@fau.de

4 Institut für Pathologie, Friedrich-Alexander Universität Erlangen-Nürnberg, Klinikum Bayreuth, Preuschwitzer Straße 101, 95445 Bayreuth, Germany

5 Bayreuther Zentrum für Molekulare Biowissenschaften (BZMB), Universität Bayreuth, Universitätsstraße 30, 95447 Bayreuth, Germany

* Correspondence: william.sterlacci@klinikum-bayreuth.de (W.S.); birgitta.woehrl@uni-bayreuth.de (B.M.W.)

+ These authors contributed equally to this work.

check for

updates

Citation: Haumaier, F.;

Schneider-Fuchs, A.; Backert, S.; Vieth, M.; Sterlacci, W.; Wöhrl, B.M. Rapid Detection of Quinolone Resistance Mutations in gyrA of Helicobacter pylori by Real-Time PCR. Pathogens 2022, 11, 59. https:// doi.org/10.3390/pathogens11010059 Academic Editors: Olivier Sparagano and David Rodriguez-Lazaro

Received: 2 December 2021 Accepted: 29 December 2021 Published: 3 January 2022

Publisher's Note: MDPI stays neutral with regard to jurisdictional claims in published maps and institutional affiliations.

Copyright: (C) 2022 by the authors. Licensee MDPI, Basel, Switzerland. This article is an open access article distributed under the terms and conditions of the Creative Commons Attribution (CC BY) license (https:// creativecommons.org/licenses/by/ $4.0 /)$.

\begin{abstract}
The treatment of infections by the gastric pathogen Helicobacter pylori (H. pylori) has become more difficult due to increased rates of resistances against various antibiotics. Typically, atriple therapy, employing a combination of at least two antibiotics and a proton pump inhibitor, is used to cure H. pylori infections. In case of first-line therapy failure, quinolones are commonly applied in a secondline therapy. To prevent second-line treatment failures, we developed an improved method to detect the most common quinolone-resistance mutations located in the quinolone-resistance-determining region (QRDR) of the bacterial gyrA gene. Biopsy material from the gastric mucosa of infected patients was used to identify quinolone-resistant strains before the onset of drug administration. Two different wild-type and six mutant QRDR sequences were included. Melting curve analyses were performed with corresponding gyrA plasmid DNAs using a real-time polymerase chain reaction (RT-PCR) assay. By applying a combination of only two different fluorescent probes, this assay allows wild-type sequences to be unambiguously distinguished from all known mutant QRDR sequences of H. pylori. Next, the $\mathrm{T}_{\mathrm{m}}$ values of patient DNAs were established, and the genotypes were confirmed by sequencing. Thus, quinolone-resistant $H$. pylori strains can be easily and quickly diagnosed before treatment, which will help to avoid the administration of ineffective drug regimes.
\end{abstract}

Keywords: Helicobacter pylori; antibiotic resistance; quinolone resistance; QRDR mutation diagnosis; FRET; gyrA; RT-PCR

\section{Introduction}

The Gram-negative bacterium Helicobacter pylori (H. pylori) infects the gastric mucosa and is associated with different human diseases, e.g., chronic active gastritis, peptic ulcer disease, gastric cancer or mucosa-associated lymphoid tissue (MALT)-lymphoma [1]. In Germany, H. pylori infections are commonly treated first-line by a triple therapy consisting of two different antibiotics, usually amoxicillin and clarithromycin or metronidazole, and a proton pump inhibitor (PPI) [2]. However, this standard first-line therapy for the eradication of $H$. pylori faces a serious problem due to the increasing rates of resistance against these antibiotics, which may result in therapy failure. Thus, a quadruple therapy consisting of bismuth in combination with amoxicillin, a PPI and a quinolone, e.g., levofloxacin, is now often recommended $[3,4]$. 
Quinolones target the DNA gyrase, which is an essential enzyme in bacterial replication that can create negative and positive supercoils in DNA by transiently introducing double-strand breaks in an ATP-dependent reaction [5]. DNA gyrase is heterotetrameric consisting of two A and two B subunits, respectively. The A subunit contains a tyrosine residue in the active center that covalently binds the newly generated $5^{\prime}$ termini of DNA during the cleavage reaction, while the $B$ subunit harbors the ATPase domain responsible for DNA cleavage and ligation. Quinolones were first reported to target the A subunit of the DNA gyrase in H. pylori [6]. However, it has been also suggested that mutations in the B subunit may contribute to quinolone resistance [7-9]. In particular, resistance against quinolones can be achieved by single point mutations in the so-called quinolone resistance-determining region (QRDR) of the gyrA gene of $H$. pylori [10-12]. The mutations conferring resistance result in specific amino acid exchanges at codons 87 and/or 91 in QRDR, which, in turn, lead to a weaker binding of the antibiotic. The most common amino acid exchanges appear to be N87K and D91G, D91N and D91Y (Table 1) [13-15]. The N87K mutation alone confers high resistance to levofloxacin and gatifloxacin [7]. Depending on the geographical region, in which the isolates were obtained, additional but rarely occurring amino acid exchanges have been detected, i.e., N87I, N87R, D91M, D91C, D91A, and $\mathrm{N} 87 \mathrm{H}$ in combination with D91M [16,17]. However, the contribution of these mutations to antibiotic resistance has not been tested in all cases.

Table 1. Sequence of wild-type and mutant codons of gyrA QRDR.

\begin{tabular}{ccc}
\hline Genotype & gyrA-Codon 87 (aa) & gyrA-Codon 91 (aa) \\
\hline WT1 & AAC (Asn) & GAT (Asp) \\
WT2 & AAT (Asn) & GAT (Asp) \\
MT1 & AAA (Lys) & GAT (Asp) \\
MT2 & AAG (Lys) & GAT (Asp) \\
MT3 & AAT (Asn) & GGT (Gly) \\
MT4 & AAC (Asn) & TAT (Tyr) \\
MT5 & AAT (Asn) & Ast Asn) \\
MT6 & AAC (Asn) & AAT (Asn)
\end{tabular}

* The corresponding amino acid (aa) is shown in parenthesis.

A common method to examine resistance to quinolone is via hybridization probes on stripes. This procedure is used in the commercial kit GenoType HelicoDR from Hain Lifesciences (Nehren, Germany). Despite the advantageous suitability of this kit for cell culture testing, it requires, in addition to a thermal cycler, an incubator and an apparatus for the visual evaluation of the colour reactions on the stripes. Moreover, this test takes more than five hours to complete. Another resistance-determining technique is the wholegenome sequencing of H. pylori via the Next Generation Sequencing (NGS) methodology. NGS is especially useful in the identification of new resistance mutations. However, this approach is very expensive and time-consuming for routine examinations [18]. Above all, for the treatment of $H$. pylori infections, it is important to be able to distinguish between wild-type (WT) and mutant (MT) QRDR sequences. These requirements can be met by an RT-PCR-based assay, which can be performed in only a few hours. Furthermore, compared to any dot blot hybridization method, the sensitivity obtained using real-time polymerase chain reaction (RT-PCR) is much higher, because DNA amplification can detect very low copy numbers of the specific DNA sequence.

In this work, we developed an assay based on melting curve analyses using RTPCR to distinguish between the known WT and the most prevalent MT QRDR sequences (Table 1) [13-15]. In contrast to previous approaches, by cultivating the bacteria to determine the resistance pattern (E-test), which usually takes about five days, genotyping with RT-PCR can provide consistent results within one day, thus saving time, money and additional biopsy procedures for culture. This method allows for the reliable identification of H. pylori strains in patients, which are resistant to quinolones using an RT-PCR assay based on Förster resonance energy transfer (FRET), and the determination of different 
melting point temperatures $\left(\mathrm{T}_{\mathrm{m}}\right)$ of fluorescently labeled probes that are complementary to the relevant QRDR sequence. The assay only requires a set of three different probes, one WT sequence and two mutant sequences to reliably determine if resistant $H$. pylori is present or not. Our method provides a rapid and cost-effective new tool for the improved detection of all prevalent resistance-associated mutations against quinolones in the QRDR and, in addition, can discriminate between MT and WT sequences. Thus, the optimal combination of antibiotics for the patient can be determined before treatment.

\section{Results and Discussion}

The aim of this study was to develop a fast and low-cost assay, which can unambiguously distinguish between $H$. pylori strains from patient biopsies that are sensitive or resistant to quinolone antibiotics. Using this strategy, inefficient treatment of patients can be avoided with savings in time, money and additional biopsy procedures for culture. A previously published assay detected gyrA mutations in H. pylori by allele-specific PCR [19]. However, this method requires the application of a set of eight different allele-specific primers. After PCR amplification, the PCR products have to be visually analyzed by agarose gel electrophoresis and are distinguished according to their size.

A RT-PCR assay, developed by Glocker and Kist [14], aimed to detect WT and MT QRDR sequences bya Förster resonance energy transfer (FRET)-based RT-PCR assay, using a labeled anchor probe in combination with a labeled mutation probe for each codon. The assay established here combines that approach with a method used for the detection of clarithromycin resistance in H. pylori, as reported by Schabereiter-Gurtner et al. [20]. Only two fluorescent probes, specific to the mutant genotypes corresponding to MT1 and MT3, respectively (Table 1), are needed, but no anchor probes, since here FRET is based on the intercalation and excitation of SYBR-Green in the amplified DNA, followed by emission via the Cyanine5 (Cy5) label of the probe (Figure 1).

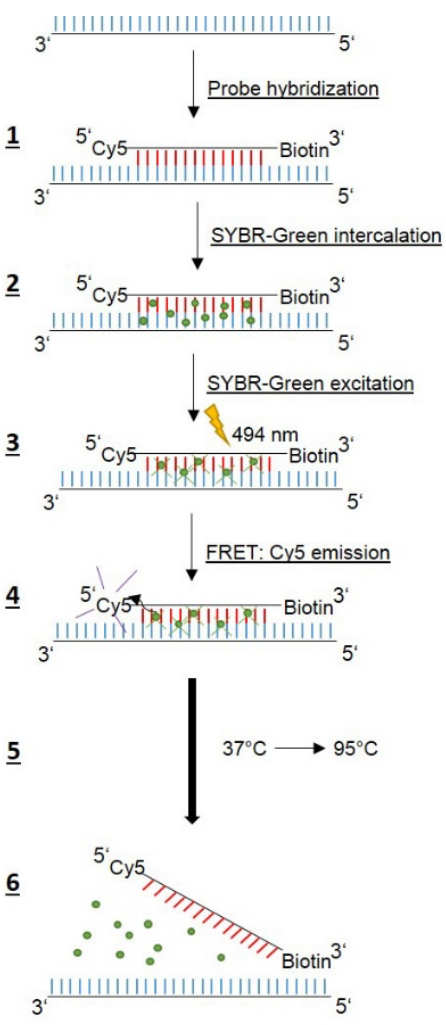

Figure 1. Schematic presentation of the sequence of events performing a melting curve analysis by RT-PCR with a Cy5 labeled hybridization probe and SYBR-green. Six individual steps were defined as indicated. For more details, see text and Tables $2-4$. 
Table 2. Cloning and mutagenesis primers.

\begin{tabular}{|c|c|}
\hline Primer & Sequence * \\
\hline FP GyrA WT1 & 5'-ATG CAT GAA TTA GGT CTT ACT-3' \\
\hline RP GyrA WT1 & 5'-TTC TTC ACT CGC CTT AGT CAT-3' \\
\hline FP GyrA WT2 & 5'-C CAC CCC CAT GGC GAT AAT GCG GTT TAT GAT GCA CTA G-3' \\
\hline RP GyrA WT2 & 5'-C TAG TGC ATC ATA AAC CGC ATT ATC GCC ATG GGG GTG G-3' \\
\hline FP GyrA MT1 & 5'-C CAC CCC CAT GGC GAT AAA GCG GTT TAT GAT GCA CTA G-3' \\
\hline RP GyrA MT1 & 5'-C TAG TGC ATC ATA AAC CGC TTT ATC GCC ATG GGG GTG G-3' \\
\hline FP GyrA MT2 & $5^{\prime}$-C CAC CCC CAT GGC GAT AAG GCG GTT TAT GAT GCA CTA G-3' \\
\hline RP GyrA MT2 & 5'-C TAG TGC ATC ATA AAC CGC CTT ATC GCC ATG GGG GTG G-3' \\
\hline FP GyrA MT3 & $5^{\prime}$-C CAC CCC CAT GGC GAT AAT GCG GTT TAT GGT GCA CTA G-3' \\
\hline RP GyrA MT3 & 5'-C TAG TGC ACC ATA AAC CGC ATT ATC GCC ATG GGG GTG G-3' \\
\hline FP GyrA MT4 & 5'-GGC GAT AAC GCG GTT TAT TAT GCA CTA GTG AGA ATG G-3' \\
\hline RP GyrA MT4 & 5'-C CAT TCT CAC GAT TGC ATA ATA AAC CGC GTT ATC GCC-3' \\
\hline FP GyrA MT5 & 5'-C CAC CCC CAT GGC GAT AAT GCG GTT TAT AAT GCA CTA G-3' \\
\hline RP GyrA MT5 & $5^{\prime}-\mathrm{C}$ TAG TGC ATT ATA AAC CGC ATT ATC GCC ATG GGG GTG G-3' \\
\hline FP GyrA MT6 & 5'-GGC GAT AAC GCG GTT TAT AAT GCA CTA GTG AGA ATG G-3' \\
\hline RP GyrA MT6 & 5'-C CAT TCT CAC GAT TGC ATT ATA AAC CGC GTT ATC GCC-3’ \\
\hline
\end{tabular}

Table 3. Sequences of hybridization probes and primers used for RT-PCR.

\begin{tabular}{cr}
\hline Oligo & \multicolumn{1}{c}{ Sequence } \\
\hline FP GyrA WT1 & 5'-ATG CAT GAA TTA GGT CTT ACT-3' $^{\prime}$ \\
RP GyrA WT1 & 5'-TTC TTC ACT CGC CTT AGT CAT-3' $^{\prime}$ \\
MT3 probe * & 5'-Cy5-ACC ATA AAC GGC ATT ATC GCC A-Biotin-3' $^{\prime}$ \\
MT1 probe & $5^{\prime}$-Cy5-ATC ATA AAC GGC TTT ATC GCC A-Biotin-3 \\
WT1 probe & $5^{\prime}$-Cy5-TGC ATC ATA AAC CGC GTT ATC G-Biotin-3' \\
\hline
\end{tabular}

* Sequence taken from [14]. The mutated bases are shown in bold.

Table 4. RT-PCR program for melting curve analysis.

\begin{tabular}{ccccc}
\hline & $\begin{array}{c}\text { Temperature } \\
\left({ }^{\circ} \mathbf{C}\right)\end{array}$ & Time (s) & $\begin{array}{c}\text { Number of } \\
\text { Cycles }\end{array}$ & $\begin{array}{c}\text { Temperature } \\
\text { Increase }\left({ }^{\circ} \mathbf{C} / \mathbf{s}\right)\end{array}$ \\
\hline Initial DNA denaturation & 95 & 600 & 1 & 4.4 \\
DNA denaturation & 95 & 5 & 70 & 4.4 \\
Hybridization & 65 & 10 & 70 & 2.2 \\
Primer elongation & 72 & 6 & 70 & 4.4 \\
Melting of double strands & 95 & 1 & 1 & 4.4 \\
Binding of probe & 37 & 1 & 1 & 2.2 \\
Melting curve & $37-95$ & 967 & 1 & 0.6 \\
Cooling & 4 & 1 & $\infty$ & 4.4 \\
\hline
\end{tabular}

To find reliable assay conditions, we first generated plasmid DNAs harboring two defined WT sequences (WT1 and WT2) and six MT QRDR sequences (MT1 to MT6) as shown in Tables 1 and 2, respectively. We decided not to use different fluorescent labels, which would allow two probes to be used at the same time to avoid misinterpretations, since all probes bind to the same region. A single-stranded DNA probe complementary to the QRDR WT1 sequence, labeled with Cy5 at the $5^{\prime}$ end and biotin at the $3^{\prime}$ end to prevent polymerization, was used for melting curve analyses (Table 3). SYBR-green, a dye intercalating into double-stranded DNA was added to the reaction mix. RT-PCR and subsequent melting curve analysis (Table 4) was performed according to the scheme shown in Figure 1. The Cy5 WT1 probe binds specifically to the region harboring codons 87 and 91 of the amplified DNA, while SYBR-green intercalates into the double-stranded DNA. The excitation of SYBR-green at $494 \mathrm{~nm}$ results in FRET to the Cy5 label, and Cy5 emission can be detected at $670 \mathrm{~nm}$. Applying a temperature-gradient $\left(37^{\circ} \mathrm{C}\right.$ to $\left.95^{\circ} \mathrm{C}\right)$ leads to dissociation of the Cy5 probe at a specific temperature and, thus, the FRET signal abates. 
Accordingly, mutations in the QRDR template DNA will give rise to lower $\mathrm{T}_{\mathrm{m}}$ values due to the weaker binding of the Cy5 probe, which is $100 \%$ complementary to only the WT1 QRDR.

Using the Cy5 WT1 probe (Table 3), corresponding to the WT1 QRDR sequence, resulted in a high background noise, which made analysis of the melting curves difficult, and no signal could be detected for MT3 (data not shown). Thus, we designed new Cy5 probes corresponding to MT1 and MT3 (Table 3) and performed RT-PCR reactions to determine the $\mathrm{T}_{\mathrm{m}}$-values. With both probes, the two WT sequences, as well as all MT QRDR sequences (Table 1), could be detected and exhibited different $T_{m}$ values (Figure 2).

A

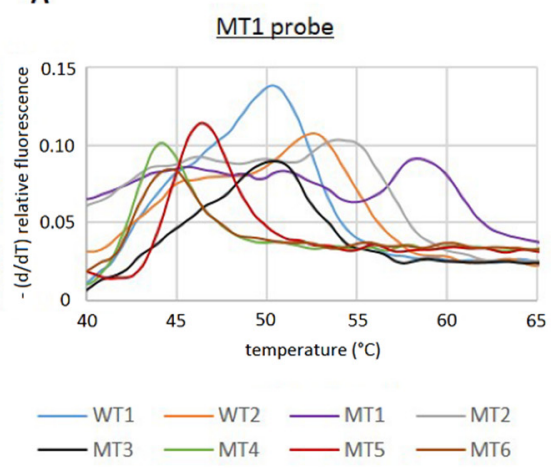

B

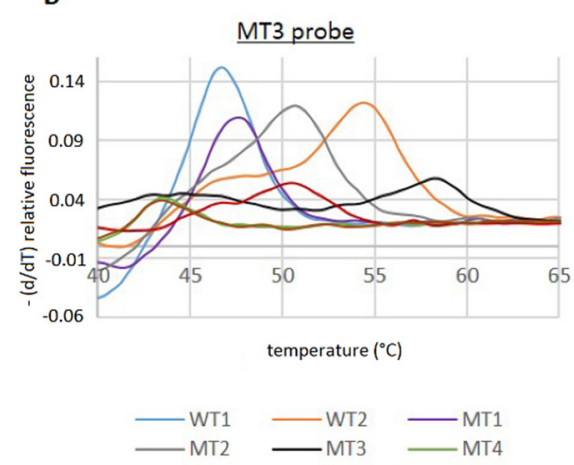

Figure 2. Melting curve analyses of WT and MT QRDR sequences by RT-PCR. Analyses were performed according to the protocol shown in Table 4 with the MT1 probe (A) or MT3 probe (B).

However, since the $T_{m}$ values of some of the MT PCR products were very similar compared to the WT, it was not possible to unambiguously distinguish between all MT and WT sequences with just one of the Cy5 probes. Using the MT3 probe, no discrimination of the $\mathrm{T}_{\mathrm{m}}$ of the WT1/MT1, MT2/MT5, and MT4/MT6 was possible. However, the MT1 probe allowed us to distinguish between the WT1 and MT1 sequence, but the discrimination of WT2/MT2, WT1/MT3, and MT4/MT6 could not be achieved (Figure 2; Table 5).

Table 5. $\mathrm{T}_{\mathrm{m}}$-values of MT1 and MT3 probes.

\begin{tabular}{ccc}
\hline Genotype & $\mathbf{T}_{\mathbf{m}}$ MT1 Probe $\left({ }^{\circ} \mathbf{C}\right)$ & $\mathbf{T}_{\mathbf{m}}$ MT3 Probe $\left({ }^{\circ} \mathbf{C}\right)$ \\
\hline WT1 & $49.99 \pm 0.184$ & $46.86 \pm 0.139$ \\
WT2 & $52.58 \pm 0.057$ & $54.79 \pm 0.625$ \\
MT1 & $58.49 \pm 0.099$ & $47.61 \pm 0.179$ \\
MT2 & $53.43 \pm 0.629$ & $50.72 \pm 0$ \\
MT3 & $50.16 \pm 0.201$ & $58.20 \pm 0.165$ \\
MT4 & $44.26 \pm 0.114$ & $43.61 \pm 0.132$ \\
MT5 & $46.42 \pm 0.120$ & $50.54 \pm 0.231$ \\
MT6 & $44.62 \pm 0.057$ & $43.59 \pm 0.206$ \\
\hline
\end{tabular}

The mean values including the standard deviation of three independent experiments are shown. The different shades of gray (dark, medium, light) indicate $\mathrm{T}_{\mathrm{m}}$ pairs that cannot be distinguished using only the MT1 or MT3 probe, respectively.

Nevertheless, our setup allowed for a general discrimination between WT and MT sequences. Since only patients infected with H. pylori WT strains, which are susceptible to quinolones, can be successfully treated with the antibiotic, it is sufficient to be able to separate the WT sequences from all MT sequences. Thus, by using our two MT Cy5 probes in parallel, it is possible to assign a sequence either to a WT or MT phenotype, respectively. If a patient sample is tested with the MT1 probe and a $\mathrm{T}_{\mathrm{m}}$ of ca. $50{ }^{\circ} \mathrm{C}$ is determined, it will not be clear whether the H. pylori strain harbors the WT1 or MT3 QRDR sequence (Table 5). Thus, a second RT-PCR reaction with the MT3 probe can be performed for identification. 
However, with the MT3 probe, the WT1 will have a $\mathrm{T}_{\mathrm{m}}$ of $46.86{ }^{\circ} \mathrm{C}$, the $\mathrm{T}_{\mathrm{m}}$ of the MT3 genotype will be increased to $58.2^{\circ} \mathrm{C}$ due to the $100 \%$ complementarity of the MT3 probe. The same procedure can be applied if $\mathrm{T}_{\mathrm{m}}=52.7^{\circ} \mathrm{C}$, and no distinction between WT2 and MT2 can be made, since the $\mathrm{T}_{\mathrm{m}}$ values of the two sequences differ by $4{ }^{\circ} \mathrm{C}$ when the MT3 probe is used. On the other hand, no distinction between WT1 and MT1 can be made if only the MT3 probe is used. However, by applying the MT1 probe, the $\mathrm{T}_{\mathrm{m}}$ values are easily distinguishable $\left(49.99^{\circ} \mathrm{C}\right.$ vs. $\left.58.49^{\circ} \mathrm{C}\right)$.

Finally, to further examine the validity of our results, we analyzed DNA isolated from biopsies taken from patients with known H. pylori infections. Each sample was tested with the MT1, as well as with the MT3 probe. In addition, to confirm the results obtained by melting curve analyses, the patient DNAs were sequenced to determine the QRDR genotype (Table 6, Figure 3). All sequencing data were in agreement with the genotype determined by RT-PCR (Table 6, Figure 3). As negative controls, and to determine the specificity of our test, we isolated biopsy DNA from six patients known not to be infected with $H$. pylori and performed RT-PCR with the QRDR primers. As expected, no QRDR sequence could be detected in all cases (Table 6, bottom, Supplementary Data, Figure S1A,B), which confirms the good specificity of this test.
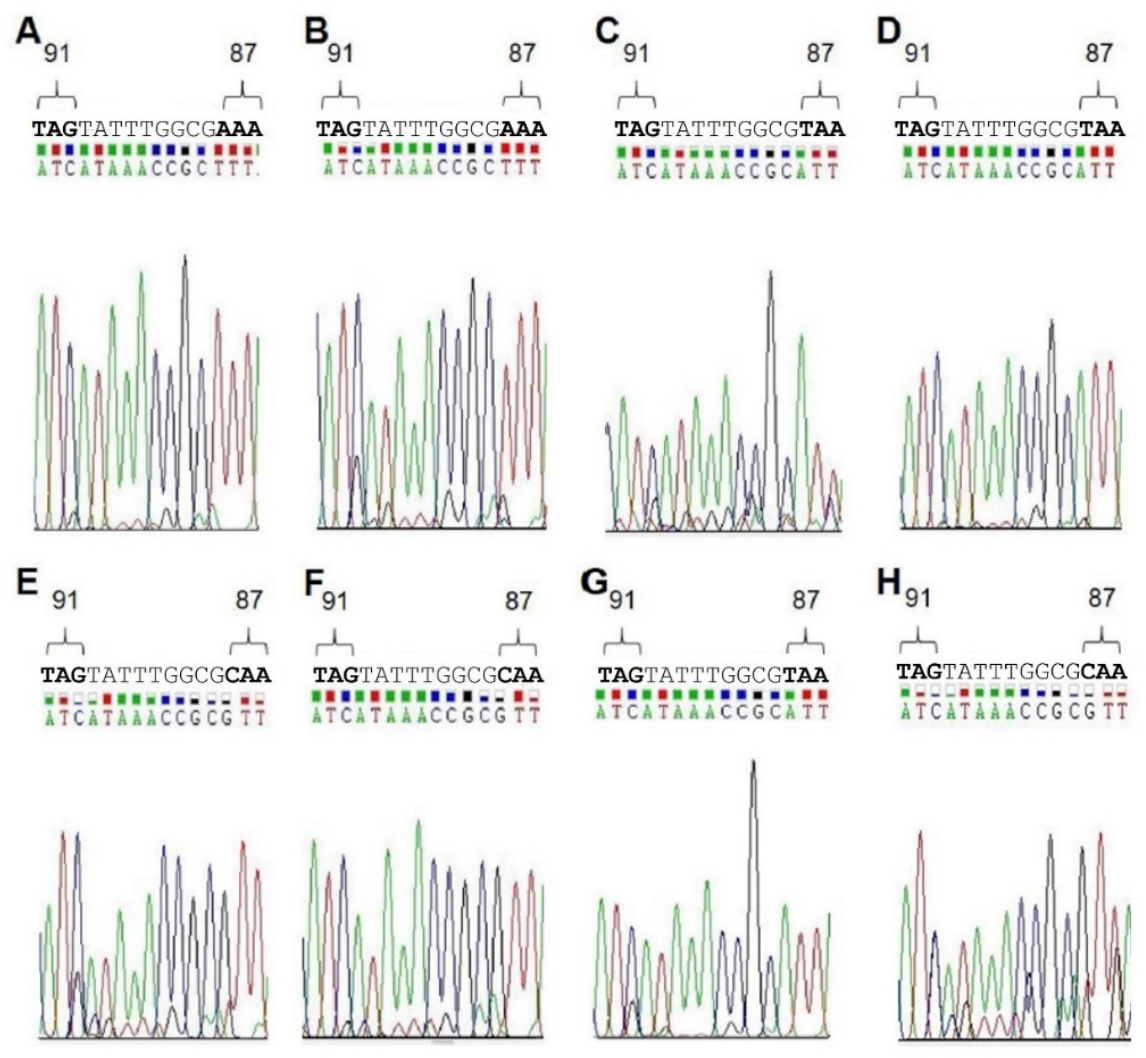

Figure 3. Sequencing data obtained from DNA in gastric biopsies of $H$. pylori-positive patients. Eight DNA samples (A-H) used for melting curve analysis were sequenced with the gyrA-RP as sequencing primer (Table 3). The relevant region of the QRDR harboring codons 87 and 91 is shown. 
Table 6. Assignment of patient DNA samples by melting curve analyses and DNA sequencing.

\begin{tabular}{|c|c|c|c|c|}
\hline DNA Sample & $\begin{array}{c}\mathrm{T}_{\mathrm{m}} \mathrm{MT1} \text { Probe } \\
\left({ }^{\circ} \mathrm{C}\right)\end{array}$ & $\begin{array}{c}\mathrm{T}_{\mathrm{m}} \text { MT3 Probe } \\
\left({ }^{\circ} \mathrm{C}\right)\end{array}$ & Assignment & $\begin{array}{c}\text { DNA } \\
\text { Sequencing }\end{array}$ \\
\hline $\mathbf{A}$ & 58.9 & 47.8 & MT1 & MT1 \\
\hline B & 59.4 & 48.8 & MT1 & MT1 \\
\hline $\mathrm{C}$ & 53.3 & 56.2 & WT2 & WT2 \\
\hline D & 53.9 & 54.7 & WT2 & WT2 \\
\hline $\mathbf{E}$ & 48.7 & 46.4 & WT1 & WT1 \\
\hline $\mathbf{F}$ & 51.5 & 48.2 & WT1 & WT1 \\
\hline G & 53.5 & 56.2 & WT2 & WT2 \\
\hline $\mathbf{H}$ & 51.5 & 47.9 & WT1 & WT1 \\
\hline controls * & / & / & / & / \\
\hline
\end{tabular}

* six patient samples without known H. pylori infection were used as controls.

\section{Material and Methods}

Plasmids. The gyrA QRDR sequence of two antibiotics-susceptible clinical WT strains were utilized, WT1 (GenBank accession no. CP026515.1) and WT2 (GenBank accession no. CP026515.1). The two WT sequences differ only in the codon at position 87 (Table 1, top). The WT1 gyrA sequence was amplified by PCR with the FP and RP GyrA WT1 primers (Table 2) using sequenced patient DNA as a template, and cloned into the vector pUC57 via the restriction sites XbaI and BamHI (Genscript, Piscataway, NJ, USA). WT2 and six QRDR mutations (named MT1 to MT6) were introduced by site-directed mutagenesis in the WT1 sequence (Table 1). The primers for mutagenesis shown in Table 2 were applied according to the Quick Change mutagenesis protocol (Agilent Technologies, Santa Clara, CA, USA) and confirmed by standard sequencing (GATC Biotech AG, Konstanz, Germany). Plasmids were propagated in Escherichia coli strains XL1-Blue or Top10 and isolated using the QIAprep Spin Miniprep Kit (Qiagen, Hilden, Germany).

Patient DNA isolation, amplification and sequencing. DNA of patients infected with H. pylori was isolated from formalin fixed, paraffin embedded (FFPE) gastric biopsies (ethics statement number: 322_21 Bc, University of Erlangen, Germany) using the Maxwell ${ }^{\circledR} 16$ LEV Blood Kit (Promega GmbH, Mannheim, Germany). Usually, six thin sections of $2 \mu \mathrm{m}$ thickness were used for DNA extractions. The concentration was measured by UV-Vis spectroscopy at $260 \mathrm{~nm}$. Approximately 200-400 $\mathrm{ng}$ of isolated DNA was used to amplify the QRDR sequence by PCR using the primers gyrA-FP and gyrA-RP (Table 3) with the PCR program depicted in Table 4 without the melting curve step. To avoid misinterpretation of the results, lower DNA concentrations are not recommended. The amplified QRDR fragments were sequenced using gyrA-RP as a sequencing primer (GATC Biotech AG, Konstanz, Germany).

Melting curve analyses. All plasmid and patient DNAs were analyzed by melting curve analyses. Primers and probes (metabion $\mathrm{GmbH}$, Planegg/Steinkirchen, Germany) used are shown in Table 3. The reaction mix contained $0.625 \mu \mathrm{M}$ forward primer $(\mathrm{FP})$, $0.19 \mu \mathrm{M}$ reverse primer (RP), $0.5 \mu \mathrm{M}$ probe, $3 \mathrm{mM} \mathrm{MgCl}_{2}, 2 \mu \mathrm{L}$ LightCycler FastStart DNA Master SYBR green I (Roche Diagnostics, Mannheim, Germany) and 40 ng of template DNA in $20 \mu \mathrm{L}$ reactions. Samples were analyzed using a Cobas Z 480 Analyzer (Roche Diagnostics, Mannheim, Germany) according to the RT PCR program shown in Table 4. The results were evaluated with the LightCycler ${ }^{\circledR} 480$ software 1.5.1 (Roche Diagnostics, Mannheim, Germany). Several independent melting curve analyses were performed for each QRDR sequence and the average of the melting temperature $\left(T_{m}\right)$, as well as the standard deviation (S) were estimated according to Equations (1) and (2):

$$
\begin{gathered}
\overline{\mathrm{T}_{\mathrm{m}}}=\frac{\left(\sum \mathrm{T}_{\mathrm{m}}\right)}{\mathrm{n}} \\
\mathrm{S}=\sqrt{\frac{\sum_{\mathrm{n}}\left(\mathrm{T}_{\mathrm{m}}-\overline{\mathrm{T}_{\mathrm{m}}}\right)^{2}}{\mathrm{n}}}
\end{gathered}
$$


$\mathrm{n}$, number of measurements; $\mathrm{T}_{\mathrm{m}}$, melting temperature; $\overline{\mathrm{T}_{\mathrm{m}}}$, average of melting temperature of $\mathrm{n}$ measurements; $\mathrm{S}$, standard deviation.

\section{Conclusions}

Our results demonstrate that the FRET-based melting curve analysis using SYBRGreen in combination with two Cy5 labeled probes provides a valuable and reasonably priced tool, since the assay can be performed by various commercially available RT-PCR instruments, which are able to detect the Cy5 signal. If necessary, the method can be extended to include rare mutations by determining the corresponding melting temperature. It can reliably distinguish between WT and MT QRDR sequences, which is necessary to efficiently treat patients with quinolones.

Supplementary Materials: The following are available online at https:/ /www.mdpi.com/article/10 .3390 / pathogens11010059/s1, Figure S1: Melting curve analyses with the MT1 and MT 3 probes and DNA from patients without $H$. pylori infection.

Author Contributions: F.H. and A.S.-F. performed the experiments. B.M.W., W.S. and M.V. designed the experiments and supervised the project. B.M.W., W.S., F.H. and A.S.-F. evaluated the data. M.V. and S.B. provided conceptual input. All authors contributed to writing the manuscript. All authors have read and agreed to the published version of the manuscript.

Funding: This work was supported by the Klinikum Bayreuth $\mathrm{GmbH}$ and the University of Bayreuth. Funded by the Deutsche Forschungsgemeinschaft (DFG, German Research Foundation)—491183248 and by the Open Access Publishing Fund of the University of Bayreuth.

Institutional Review Board Statement: The study was conducted in accordance with the Declaration of Helsinki, and approved by the Ethics Committee of the Friedrich-Alexander Universität ErlangenNürnberg, protocol code: 322_21 Bc; 17 August 2021.

Informed Consent Statement: Upon admission to the hospital, all patients signed an informed consent.

Data Availability Statement: Data were taken from the archive of the Institute of Pathology and stored there according to national law. Data for this study were anonymised in accordance with data protection laws and local regulations.

Acknowledgments: We thank Ulrike Persau for excellent technical assistance and Laura Debbert for performing initial PCR experiments.

Conflicts of Interest: The authors declare no conflict of interest.

\section{Abbreviations}

$\begin{array}{ll}\text { Abbreviation } & \text { Full Name } \\ \text { Cy5 } & \text { Cyanine5 } \\ \text { FRET } & \text { Förster resonance energy transfer } \\ \text { FP } & \text { forward primer } \\ \text { H. pylori } & \text { Helicobacter pylori } \\ \text { MT } & \text { mutant } \\ \text { QRDR } & \text { Quinolone-resistance-determining region } \\ \text { RP } & \text { reverse primer } \\ \text { RT-PCR } & \text { real-time polymerase chain reaction } \\ \mathrm{T}_{\mathrm{m}} & \text { melting point } \\ \text { WT } & \text { wild-type }\end{array}$

\section{References}

1. Suerbaum, S.; Michetti, P. Helicobacter Pylori Infection. N. Engl. J. Med. 2002, 347, 1175-1186. [CrossRef] [PubMed]

2. Malfertheiner, P.; Mégraud, F.; O'Morain, C.; Hungin, A.P.S.; Jones, R.; Axon, A.; Graham, D.Y.; Tytgat, G. European Helicobacter Pylori Study Group (EHPSG) Current Concepts in the Management of Helicobacter Pylori Infection-the Maastricht 2-2000 Consensus Report. Aliment Pharmacol. Ther. 2002, 16, 167-180. [CrossRef] [PubMed] 
3. Malfertheiner, P.; Megraud, F.; O'Morain, C.A.; Atherton, J.; Axon, A.T.R.; Bazzoli, F.; Gensini, G.F.; Gisbert, J.P.; Graham, D.Y.; Rokkas, T.; et al. Management of Helicobacter Pylori Infection-the Maastricht IV/Florence Consensus Report. Gut 2012, 61, 646-664. [CrossRef] [PubMed]

4. Malfertheiner, P.; Megraud, F.; O’Morain, C.A.; Gisbert, J.P.; Kuipers, E.J.; Axon, A.T.; Bazzoli, F.; Gasbarrini, A.; Atherton, J.; Graham, D.Y.; et al. Management of Helicobacter Pylori Infection-the Maastricht V/Florence Consensus Report. Gut 2017, 66, 6-30. [CrossRef] [PubMed]

5. Kampranis, S.C.; Bates, A.D.; Maxwell, A. A Model for the Mechanism of Strand Passage by DNA Gyrase. Proc. Natl. Acad. Sci. USA 1999, 96, 8414-8419. [CrossRef] [PubMed]

6. Tankovic, J.; Lascols, C.; Sculo, Q.; Petit, J.-C.; Soussy, C.-J. Single and Double Mutations in GyrA but Not in GyrB Are Associated with Low- and High-Level Fluoroquinolone Resistance in Helicobacter Pylori. Antimicrob. Agents Chemother. 2003, 47, 3942-3944. [CrossRef] [PubMed]

7. Rimbara, E.; Noguchi, N.; Kawai, T.; Sasatsu, M. Fluoroquinolone Resistance in Helicobacter Pylori: Role of Mutations at Position 87 and 91 of GyrA on the Level of Resistance and Identification of a Resistance Conferring Mutation in GyrB. Helicobacter 2012, 17, 36-42. [CrossRef]

8. Miyachi, H.; Miki, I.; Aoyama, N.; Shirasaka, D.; Matsumoto, Y.; Toyoda, M.; Mitani, T.; Morita, Y.; Tamura, T.; Kinoshita, S.; et al. Primary Levofloxacin Resistance and GyrA/B Mutations among Helicobacter Pylori in Japan. Helicobacter 2006, 11, $243-249$. [CrossRef]

9. Miftahussurur, M.; Shrestha, P.K.; Subsomwong, P.; Sharma, R.P.; Yamaoka, Y. Emerging Helicobacter Pylori Levofloxacin Resistance and Novel Genetic Mutation in Nepal. BMC Microbiol. 2016, 16, 256. [CrossRef] [PubMed]

10. Drlica, K.; Zhao, X. DNA Gyrase, Topoisomerase IV, and the 4-Quinolones. Microbiol. Mol. Biol. Rev. 1997, 61, 377-392. [CrossRef] [PubMed]

11. Moore, R.A.; Beckthold, B.; Wong, S.; Kureishi, A.; Bryan, L.E. Nucleotide Sequence of the GyrA Gene and Characterization of Ciprofloxacin-Resistant Mutants of Helicobacter Pylori. Antimicrob. Agents Chemother. 1995, 39, 107-111. [CrossRef] [PubMed]

12. Wang, G.; Wilson, T.J.; Jiang, Q.; Taylor, D.E. Spontaneous Mutations That Confer Antibiotic Resistance in Helicobacter Pylori. Antimicrob. Agents Chemother. 2001, 45, 727-733. [CrossRef] [PubMed]

13. Nishizawa, T.; Suzuki, H.; Kurabayashi, K.; Masaoka, T.; Muraoka, H.; Mori, M.; Iwasaki, E.; Kobayashi, I.; Hibi, T. Gatifloxacin Resistance and Mutations in Gyra after Unsuccessful Helicobacter Pylori Eradication in Japan. Antimicrob. Agents Chemother. 2006, 50, 1538-1540. [CrossRef] [PubMed]

14. Glocker, E.; Kist, M. Rapid Detection of Point Mutations in the GyrA Gene of Helicobacter Pylori Conferring Resistance to Ciprofloxacin by a Fluorescence Resonance Energy Transfer-Based Real-Time PCR Approach. J. Clin. Microbiol. 2004, 42, 2241-2246. [CrossRef] [PubMed]

15. Zerbetto De Palma, G.; Mendiondo, N.; Wonaga, A.; Viola, L.; Ibarra, D.; Campitelli, E.; Salim, N.; Corti, R.; Goldman, C.; Catalano, M. Occurrence of Mutations in the Antimicrobial Target Genes Related to Levofloxacin, Clarithromycin, and Amoxicillin Resistance in Helicobacter Pylori Isolates from Buenos Aires City. Microb. Drug Resist. 2017, 23, 351-358. [CrossRef] [PubMed]

16. Ontsira Ngoyi, E.N.; Atipo Ibara, B.I.; Moyen, R.; Ahoui Apendi, P.C.; Ibara, J.R.; Obengui, O.; Ossibi Ibara, R.B.; Nguimbi, E.; Niama, R.F.; Ouamba, J.M.; et al. Molecular Detection of Helicobacter Pylori and Its Antimicrobial Resistance in Brazzaville, Congo. Helicobacter 2015, 20, 316-320. [CrossRef] [PubMed]

17. Lee, J.W.; Kim, N.; Nam, R.H.; Park, J.H.; Kim, J.M.; Jung, H.C.; Song, I.S. Mutations of Helicobacter Pylori Associated with Fluoroquinolone Resistance in Korea. Helicobacter 2011, 16. [CrossRef] [PubMed]

18. Saracino, I.M.; Pavoni, M.; Zullo, A.; Fiorini, G.; Lazzarotto, T.; Borghi, C.; Vaira, D. Next Generation Sequencing for the Prediction of the Antibiotic Resistance in Helicobacter Pylori: A Literature Review. Antibiotics 2021, 10, 437. [CrossRef] [PubMed]

19. Nishizawa, T.; Suzuki, H.; Umezawa, A.; Muraoka, H.; Iwasaki, E.; Masaoka, T.; Kobayashi, I.; Hibi, T. Rapid Detection of Point Mutations Conferring Resistance to Fluoroquinolone in GyrA of Helicobacter Pylori by Allele-Specific PCR. J. Clin. Microbiol. 2007, 45, 303-305. [CrossRef] [PubMed]

20. Schabereiter-Gurtner, C.; Hirschl, A.M.; Dragosics, B.; Hufnagl, P.; Puz, S.; Kovách, Z.; Rotter, M.; Makristathis, A. Novel Real-Time PCR Assay for Detection of Helicobacter Pylori Infection and Simultaneous Clarithromycin Susceptibility Testing of Stool and Biopsy Specimens. J. Clin. Microbiol. 2004, 42, 4512-4518. [CrossRef] [PubMed] 